University of Wollongong

Research Online

Australian Institute for Innovative Materials -

Papers

Australian Institute for Innovative Materials

$1-1-2016$

\title{
Magnetic field actuated manipulation and transfer of oil droplets on a stable underwater superoleophobic surface
}

\author{
Haifeng Feng \\ University of Wollongong, hf533@uowmail.edu.au \\ Xun Xu \\ University of Wollongong, xun@uow.edu.au \\ Weichang Hao \\ Beihang University, whao@buaa.edu.cn \\ Yi Du \\ University of Wollongong, ydu@uow.edu.au \\ Dongliang Tian \\ University of Wollongong, dtian@uow.edu.au
}

See next page for additional authors

Follow this and additional works at: https://ro.uow.edu.au/aiimpapers

Part of the Engineering Commons, and the Physical Sciences and Mathematics Commons

Research Online is the open access institutional repository for the University of Wollongong. For further information contact the UOW Library: research-pubs@uow.edu.au 


\title{
Magnetic field actuated manipulation and transfer of oil droplets on a stable underwater superoleophobic surface
}

\author{
Abstract \\ The transport of fluids at functional interfaces, driven by the external stimuli, is well established. The \\ lossless transport of oil-based fluids under water remains a challenge, however, due to their high \\ stickiness towards the surface. Here, a superhydrophilic and underwater superoleophobic tri-phase \\ water/oil/solid nanoarray surface has been designed and prepared. The unique tri-phase surface exhibits \\ underwater superoleophobic properties with an extremely low stickiness towards oil-based fluids. The \\ magnetic-field-driven manipulation and transport of oil-based magnetic fluids are demonstrated under \\ water, which opens up a new pathway to design flexible and smart devices for the control and transfer of \\ liquid droplets by using tri-phase systems.

\section{Disciplines} \\ Engineering | Physical Sciences and Mathematics

\section{Publication Details} \\ Feng, H., Xu, X., Hao, W., Du, Y., Tian, D. \& Jiang, L. (2016). Magnetic field actuated manipulation and \\ transfer of oil droplets on a stable underwater superoleophobic surface. Physical Chemistry Chemical \\ Physics, 18 (24), 16202-16207.

\section{Authors} \\ Haifeng Feng, Xun Xu, Weichang Hao, Yi Du, Dongliang Tian, and Lei Jiang
}




\title{
Magnetic field actuated manipulation and transfer of oil droplets on a stable underwater superoleophobic surface
}

\author{
Haifeng Feng, ${ }^{a, b}$ Xun $\mathrm{Xu}^{\mathrm{a}, \mathrm{b}}$ Weichang Hao, ${ }^{\mathrm{b}, \mathrm{c}} \mathrm{Yi} \mathrm{Du}{ }^{{ }^{a}, \mathrm{~b}}$ Dongliang Tian ${ }^{* a, b, d}$ and Lei Jiang ${ }^{\mathrm{b}, \mathrm{d}, \mathrm{e}}$ \\ The transport of fluids at functional interfaces, driven by the external stimuli, is well established. Lossless transport of oil- \\ based fluids underwater remains a challenge, however, due to their high stickiness towards the surface. Here, a \\ superhydrophilic and underwater superoleophobic tri-phase water/oil/solid nanoarray surface has been designed and \\ prepared. The unique tri-phase surface exhibits an underwater superoleophobic property with an extremely low stickiness \\ towards oil-based fluids. The magnetic-field-driven manipulation and transport of oil-based magnetic fluids are \\ demonstrated underwater, which opens up a new pathway to design flexibility and smart devices for control and transfer \\ of liquid droplets by using tri-phase systems.
}

\section{Introduction,}

Controllable manipulation and transfer of small amounts of liquid droplets in a medium is crucial in miniature systems for pharmaceutics and for chemical, medical, and biological applications, which have been explored extensively for decades. Recent studies on functional nano/microchannel structures have demonstrated excellent strategies for design flexibility and smart devices for the control and transfer of liquid droplets. ${ }^{1-6}$ External actuating forces, including pressure differences, electric fields, thermal gradients, and acoustic stimulation, have been applied in nano/microchannel fluidic devices to control the movement of liquid droplets. ${ }^{7-13}$ It has been successfully demonstrated that the droplets tend to move free of loss if superhydrophobic (to water-based liquid) or superoleophobic (to oil-based liquid) surfaces are used in air. ${ }^{14-20}$ Nevertheless, manipulation of liquid droplets in a liquid medium remains a big challenge so far for multiple-phase contact modes. For example, although oil generally exhibits superoleophobic behaviour on solid substrates in water, it is extremely challenging to achieve the controllable transfer of oil underwater due to the difficulty of coupling actuating fields in a liquid medium. In addition, it is difficult to manipulate discrete oil droplets in a manner that is free of loss due to their high stickiness to a solid surface underwater.

In a multiphase interface, the wettability can be tuned by any phase involved in the system and, hence, an adjustable wettability is facilitated in various environments. For example, fish scales can be self-cleaning due to their oil-repellent nature underwater. $^{20}$ Artificial tri-phase (solid-oil-water and solidwater-air) interfaces that mimic fish scales were fabricated in order to achieve a superoleophobic and/or superhydrophobic surface in the liquid medium. ${ }^{21-24}$ Nevertheless, the adhesive hysteresis hinders the lossless transport of liquids in another liquid medium.

Herein, we have designed and fabricated a tri-phase water/oil/solid nanoarray interface that shows non-sticking superoleophobicity underwater. By incorporating external magnetic actuation, non-contact controllable manipulation of oil-based magnetic fluids on such interfaces is realized. We demonstrate that a magnetic fluid can be used to collect and transfer oil droplets on this interface, driven by an external magnetic field underwater. The unique tri-phase interface is important for maintaining the stability of the superoleophobic surface, and is crucial for freely controlling oil droplets on such a surface in water. This work is promising for the application of controllable transfer and collection of liquid cargo by using magnetic fluid in water through tuneable magnetic actuation.

\section{Experimental}

ZnO nanorod array samples were fabricated by a lowtemperature hydrothermal method. The substrates used to grow ZnO nanorods were clean glass. ZnO seed layers were produced by spin-coating zinc acetate dehydrate ethanol solution $(0.01 \mathrm{M})$ on the glass substrates. The hydrothermal reaction in an aqueous solution was prepared by mixing $\mathrm{Zn}\left(\mathrm{NO}_{3}\right)_{2}(0.05 \mathrm{M})$, ammonia, and urea. The density and particle size of $\mathrm{ZnO}$ can be precisely controlled by adjusting the concentration of ammonia and urea. The sealed Teflon-lined stainless steel autoclave was kept at $90{ }^{\circ} \mathrm{C}$ for $4 \mathrm{~h}$. Then, the ZnO nanorod arrays samples were washed with deionized water several times and dried in air at room temperature for $24 \mathrm{~h}$.

The $\mathrm{ZnO}$ smooth film was synthesized by a photochemical reaction. The glass substrates were cleaned extensively and then dipped in $5 \%$ poly(diallyldimethylammonium chloride) aqueous solution for $30 \mathrm{~min}$. The cationic solution, $\mathrm{ZnO}_{2}$ sol, was prepared by mixing $\mathrm{Zn}\left(\mathrm{NO}_{3}\right)_{2}$ aqueous solution $(10 \mathrm{~g} / \mathrm{L}$, $100 \mathrm{~mL}$ ) and $2 \mathrm{~mL} \mathrm{H}_{2} \mathrm{O}_{2}$ under light irradiation for $3 \mathrm{~h}$. Poly(sodium-p-styrenesulfonate) ( $2 \mathrm{~g} / \mathrm{L}$ ) was adopted as the anionic solution. The modified glass substrates were alternately immersed in the anionic solution and then the cationic solution. The substrates were rinsed intensively with water and dried with nitrogen between the consecutive immersions. The $\mathrm{ZnO}$ smooth films were obtained by annealing the as-prepared substrates at $550{ }^{\circ} \mathrm{C}$ for $2 \mathrm{~h}$.

Characterizations of the manipulation and transfer of oil droplets on the $\mathrm{ZnO}$ nanorod arrays and the $\mathrm{ZnO}$ smooth films were carried out underwater. The magnetic fluid used in this work was prepared as in a previous report. ${ }^{25}$ The intensity of the external magnetic field used to manipulate and transfer the magnetic droplets was up to $42 \mathrm{mT}$. Scanning electron microscope (SEM) images were obtained with a JEOL JSM7500FA at $10 \mathrm{kV}$. A Dataphysics OCA20 CA system was used to 
measure the contact angles (CAs) at ambient temperature. At least five different CAs at different positions were measured and averaged for the same sample.

\section{Results and discussion}

Fig. $1 \mathrm{a}$ and $\mathrm{b}$ present SEM images of as-prepared $\mathrm{ZnO}$ nanoarrays. It is revealed that the $\mathrm{ZnO}$ nanoarrays consist of highly aligned uniform nanorods. The diameters of the $\mathrm{ZnO}$ nanorods are about $150 \mathrm{~nm}$ to $250 \mathrm{~nm}$, and the length is around $6 \mu \mathrm{m}$. After ultraviolet (UV, $365 \mathrm{~nm}$ ) irradiation, the asprepared $\mathrm{ZnO}$ nanoarrays exhibit superhydrophilic $\left(6^{\circ}\right)$ and superoleophilic $\left(9^{\circ}\right)$ characteristics in air, respectively, as shown in Fig. 1c and d. ${ }^{26-28}$ When a $\mathrm{ZnO}$ nanoarrays surface is placed in water, water enter the interspace of as-prepared ZnO nanorod arrays completely, which will consequently improve the repellent force to oil. ${ }^{29-31}$ Thus, the superhydrophilic surface in air shows a superoleophobic property underwater with a CA of $156^{\circ}$, as shown in the bottom panel in Fig. 1e. This underwater superoleophobic property has been investigated in previous works. ${ }^{18,32}$ In a water/air/solid system, contact angle of water on a rough surface composed of solid and air can be described by Eqn (1):

$$
\cos \theta=f \cos \theta+f-1
$$

where $\theta$ is the contact angle of a water droplet on a rough solid in air, $\theta$ is the contact angle of a water droplet on a smooth surface in air, $f$ is the area fraction of the solid on the surface.

Similarly, in an oil/water/solid system, the contact angle of oil on a rough surface composed of solid and water can be described as Eqn (2):

$$
\cos \theta_{a}^{\prime}=f \cos \theta_{a}+f-1
$$

where $\theta_{a}{ }^{\prime}$ is the contact angle of an oil droplet on a rough solid in water, $\theta_{\mathrm{a}}$ is the contact angle of an oil droplet on a smooth surface in water, $f$ is the area fraction of the solid on the surface. Therefore, compared with $\mathrm{ZnO}$ smooth film, by dramatically decreasing the area fraction of the solid area on the surface ZnO nanorod arrays, the underwater superoleophobic surface can be obtained.

Dynamic wettability characterizations of the as-prepared aligned nanorod arrays were conducted to demonstrate the surface characteristic of underwater superoleophobicity. In comparison, the same experiments were also carried out on a $\mathrm{ZnO}$ smooth film with a lower roughness than for the $\mathrm{ZnO}$ nanorod arrays. Although both the $\mathrm{ZnO}$ nanorod arrays and the smooth films clearly exhibit superoleophobicity underwater, their adhesion behaviours towards oil are distinct. As shown in Fig. 2, an oil droplet easily rolls on the nanorod arrays surface underwater with a small sliding angle of less than $5^{\circ}$ (Fig. $2 \mathrm{~b}$ and c). In contrast, the oil droplet sticks on the ZnO smooth film and does not slide, even at a much higher sliding angle (up to $15^{\circ}$ ) (Fig. $2 \mathrm{e}$ and f). As mentioned above, the interspaces of the superhydrophilic $\mathrm{ZnO}$ nanorod arrays are fully encapsulated by water when they are immersed in water, which creates a repellent force towards oil droplets and changes the surface to superoleophobic underwater. Therefore, only the top surfaces of these nanorods are in contact with the oil droplet. This particular contact mode enables the contact area between the $\mathrm{ZnO}$ nanorods and the oil droplets to become extremely small, and forms a discontinuous tri-phase (oil/water/ZnO nanorods) contact line, as illustrated by the red dotted line in Fig. $2 \mathrm{~g}$. For smooth $\mathrm{ZnO}$, on the other hand, the contact mode of the tri-phase is a continuous contact line, as marked in blue in Fig. $2 \mathrm{~h}$, which results in a larger contact area between the substrate and the oil droplets than for the $\mathrm{ZnO}$ nanorod arrays. Thus, compared with $\mathrm{ZnO}$ smooth films, the large repellent force towards oil droplets and the discontinuous tri-phase contact mode of the aligned ZnO nanoarrays surface significantly reduces the stickiness between oil droplets and the surface.

The aligned ZnO nanoarrays were then used for manipulating and transferring oil droplets underwater. To achieve the tuneable control of oil droplets on the superoleophobic surface in water by magnetic field, magnetic fluids consisting of superparamagnetic $\mathrm{Fe}_{3} \mathrm{O}_{4}$ nanoparticles were selected. The $\mathrm{Fe}_{3} \mathrm{O}_{4}$ nanoparticles were around $25 \mathrm{~nm}$ in diameter, and they could be quickly magnetized or demagnetized with/without external magnetic field. Transport of the magnetic liquid was studied on the surface of the underwater superoleophobic $\mathrm{ZnO}$ nanorod arrays and on smooth ZnO film, respectively. As shown in Fig. 3a, the top and the bottom substrates are superoleophobic $\mathrm{ZnO}$ nanorod arrays coated on glass with water filling the interspaces. A magnetic fluid droplet was injected into the interspace. A periodic external magnetic field with a frequency of $20 \mathrm{~Hz}$ was applied as the actuating force. The magnetic fluid droplet exhibited high mobility and quick response to the magnetic stimuli on the ZnO nanorod arrays. No adhesion of the magnetic fluid was observed in the transport process, which supports the low stickiness of the magnetic fluid towards the $\mathrm{ZnO}$ nanorod arrays. Whereas, as shown in Fig. $3 \mathrm{~b}$, when the top and the bottom substrates are smooth $\mathrm{ZnO}$ film coated glass, obvious delays in the movement and loss of magnetic fluid from the droplet were observed during the motion between the two substrates. Losses of weight of the magnetic liquid droplets were reflected by the change in volume of the magnetic liquid droplets on the bottom substrates without external magnetic field. As shown in Fig. 3c, after 10 transport cycles of the magnetic liquid droplets, no loss of volume was achieved between the aligned $\mathrm{ZnO}$ nanorod arrays substrates. In contrast, the smooth ZnO-film-coated glass substrates exhibited poor performance, with about $40 \%$ of the magnetic liquid droplet adhering to the top substrate after 10 cycles. The high stickiness of the smooth ZnO film without the discontinuous tri-phase contact mode is responsible for the high adhesion.

Finally, we demonstrated that an oil droplet can be collected underwater by manipulating the motion of magnetic liquid droplets by external magnetic field. As shown in Fig. 4a$\mathrm{f}$, glass substrates with aligned $\mathrm{ZnO}$ nanorod arrays coated on the surface were used as the top and bottom interfaces with water filling the interspaces. An oleophilic point was created on the bottom substrate, which is marked by a red triangle, to immobilize the oil droplet. In Fig. 4a, the magnetic fluid droplet and a colourless oil droplet were injected separately onto the bottom substrate. When an external magnetic field was set up 
on the top substrate (Fig. 4b), the magnetic fluid droplet was rapidly magnetized and drawn towards the top substrate. It was then transferred from the left to the right side by the magnetic field (Fig. 4c). Once the external magnetic field was withdrawn, the magnetic fluid became demagnetized and fell down to the underlying interface due to gravity. Then the magnetic fluid dissolved into the oil droplet (Fig. 4d). It was found that the large mixed magnetic oil droplet was also stable on the bottom $\mathrm{ZnO}$ nanorod array interface. After that, as shown in Fig. 4e-f, the mixed large magnetic droplet was transferred back to its original position driven by the external magnetic field. No losses from the oil and magnetic fluid droplets were observed during the manipulation and movement. A variety of oils are adopted in above experiments. It is demonstrated that oils, like 1, 2-dichloroethane and chloroform with high densities, can be easily controlled and manipulated on the $\mathrm{ZnO}$ nanorod arrays underwater. Meanwhile, to control and manipulate oils with densities less than water, the external magnetic field should be applied at the bottom of the equipment.

\section{Conclusions}

In summary, the above experiments achieved the free transfer and manipulation of oil droplets under water with the assistance of a superparamagnetic magnetic fluid under external magnetic field as the actuating force. It is suggested that the superoleophobic property and small adhesion towards oil are imparted to the substrate by using the specially designed ZnO nanorod arrays as the surface, the stable triphase contact mode of which can effectively decrease the stickiness between the substrate surface and the oil droplet, and prevent the loss of the oil droplet during the movement and transfer. This work is promising for the application of controllable transfer and collection of liquid cargo via an external magnetic fluid in a liquid medium through tuning magnetic actuation. In addition, the magnetic actuation mode presents huge advantages towards liquid media due to its large and long-range forces and weak interaction with nonmagnetic actuation liquid media, as well as its easy implementation conditions and low cost.

\section{Acknowledgements}

This authors are grateful for financial support from the Australian Research Council (ARC) through a Discovery Project (DP 140102581), the 973 Program (2013CB933004), the National Natural Science Foundation of China (21373001, 21421061, 51472016 and 51272015), the Youth Talent Plan of Beijing City (29201492), the China Scholarship Council (201306025005), the Fundamental Research Funds for the Central Universities (YWF-15-HHXY-012), the Key Research Program of the Chinese Academy of Sciences (KJZD-EW-M03) and the 111 project (B14009). The authors thank Dr. T. Silver for her valuable comments on this work.

\section{References}

1 A. A. Darhuber, J. P. Valentino, J. M. Davis and S. M. Troian, Appl. Phys. Lett., 2003, 82, 657.

2 O. D. Velev, B. G. Prevo and K. H. Bhatt, Nature, 2003, 426, 515.

3 J. Yong, Q. Yang, F. Chen, H. Bian, G. Du, U. Farooq and X. Hou, Adv. Mater. Interfaces, 2015, 2, 1400388.

4 S. Daniel, M. K. Chaudhury and P.-G. D. Gennes, Langmuir, 2005, 21, 4240.

5 R. Yamada and H. Tada, Langmuir, 2005, 21, 4254.

6 Y. Zhao, J. Fang, H. Wang, X. Wang and T. Lin, Adv. Mater., 2010, 22, 707.

7 S. W. Jones, O. M. Thomas and H. Aref, J. Fluid Mech., 1989, 209, 335.

8 H. A. Stone, A. D. Stroock and A. Ajdari, Annu. Rev. Fluid Mech., 2004, 36, 381.

9 W. C. Nelson and C.-J. Kim, J. Adhesion Sci. Technol., 2012, 26, 1747.

10 A. A. Darhuber, J. M. Davis and S. M. Troian, Appl. Fluids, 2003, 15, 1295.

11 A. A. Darhuber, J. P. Valentino. S. M. Troian and S. Wagner, J. Microelectromech. Syst., 2003, 12, 873.

12 F. Brochard, Langmuir, 1989, 5, 432.

13 Y. N. Cheung, N. T. Nguyen and T. N. Wong, Soft Matter, 2014, 10, 8122.

14 G. Fang, W. Li, X. Wang and G. Qiao, Langmuir, 2008, 24, 11651.

15 M. Ma, Y. Mao, M. Gupta, K. K. Gleason and G. C. Rutledge, Macromolecules, 2005, 38, 9742.

16 X. Hong, X. Gao and L. Jiang, J. Am. Chem. Soc., 2007, 129, 1478.

17 X. Yao, J. Gao, Y. Song and L. Jiang, Adv. Funct. Mater., 2011, 21, 4270 .

18 M. Liu, S. Wang, Z. Wei, Y. Song and L. Jiang, Adv. Mater., 2009, 21, 665.

19 T. Huang, L. Zhang, H. Chen and C. Gao, J. Mater. Chem. A, 2015, 3, 19517.

20 M. Sun, Y. Du, W. Hao, H. Xu, Y. Yu and T. Wang, J. Mater. Sci. Technol., 2009, 25, 53.

21 K. Liu and L. Jiang, Nano Today, 2011, 6, 155.

22 X. Zheng, Z. Guo, D. Tian, X. Zhang, W. Li and L. Jiang, ACS Appl. Mater. Interfaces, 2015, 7, 4336.

23 B. Wang, W. Liang, Z. Guo and W. Liu, Chem. Soc. Rev., 2015, 44, 336.

24 Y. C. Jung and B. Bhushan, Langmuir, 2009, 25, 14165.

25 S. Wang, C. Yang and X. Bian, J. Magn. Magn. Mater., 2012, 324, 3361.

26 X. Feng, L. Feng, M. Jin, J. Zhai, L. Jiang and D. Zhu, J. Am. Chem. Soc., 2004, 126, 62.

27 B. Cao, Y. Li, G. Duan and W. Cai, Crystal Growth \& Design, 2006, 6, 1091.

28 B. Sun and H. Sirringhaus, J. Am. Chem. Soc., 2006, 128, 16231.

29 D. Tian, X. Zhang, Y. Tian, Y. Wu, X. Wang, J. Zhai and L. Jiang, J. Mater. Chem., 2012, 22, 19652.

30 D. Tian, Z. Guo, Y. Wang, W. Li, X. Zhang, J. Zhai and L. Jiang, Adv. Funct. Mater., 2014, 24, 536.

31 D. Tian, X. Zhang, J. Zhai and L. Jiang, Phys. Chem. Chem. Phys., 2011, 13, 14606.

32 Y. Tian, B. Su and L. Jiang, Adv. Mater., 2014, 26, 6872. 

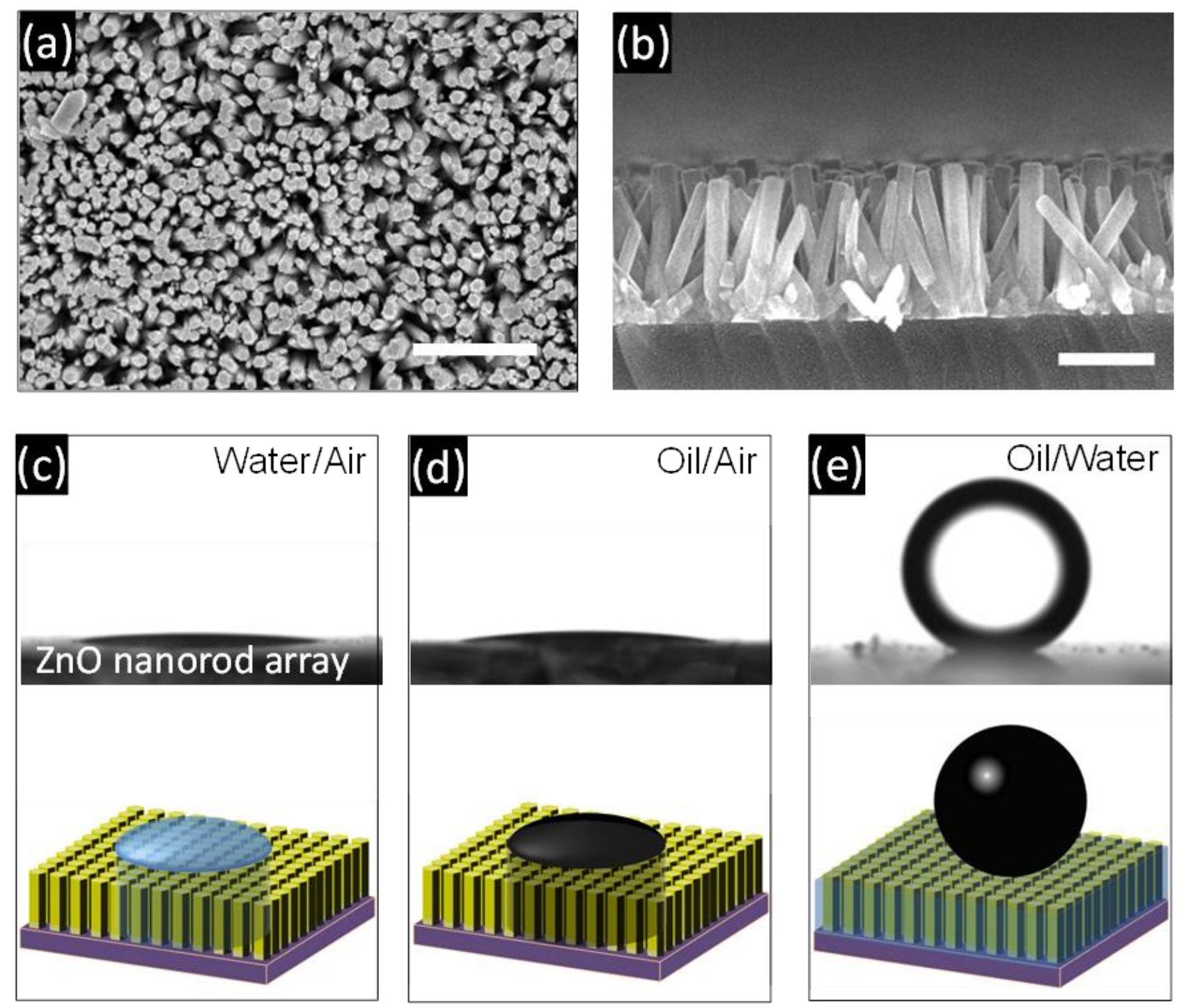

Fig. 1 Morphology and wettability characterization of the as-prepared aligned nanorod arrays. Scanning electron microscope (SEM) images of the aligned ZnO nanorod arrays: (a) Top view; (b) Cross-sectional view. The scale bars in (a) and (b) are $5 \mu \mathrm{m}$ and $2 \mu \mathrm{m}$, respectively. Photographs of water (c, top) and oil (d, top) droplets on aligned nanorod array surfaces in air. (e, top) Photograph of an oil droplet on an aligned nanorod arrays surface in water. The images at the bottoms of (c-e) are the corresponding schematic diagrams of the wetting state of water/oil on the aligned nanorod arrays in air/water, respectively. 

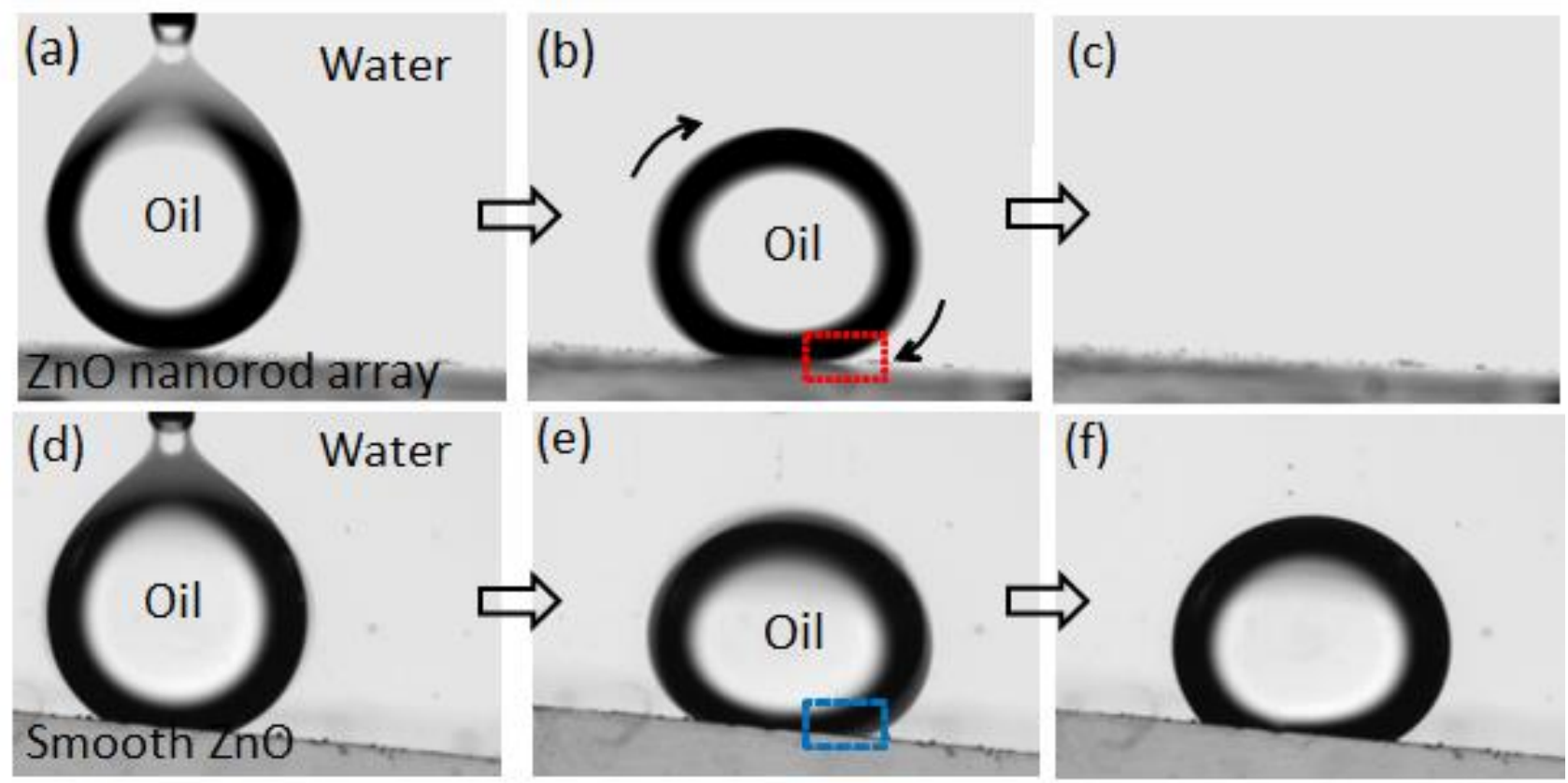

(g)

(e)

(f)

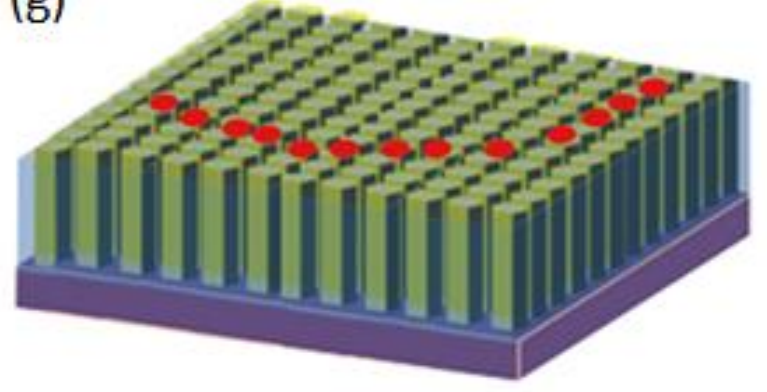

(h)

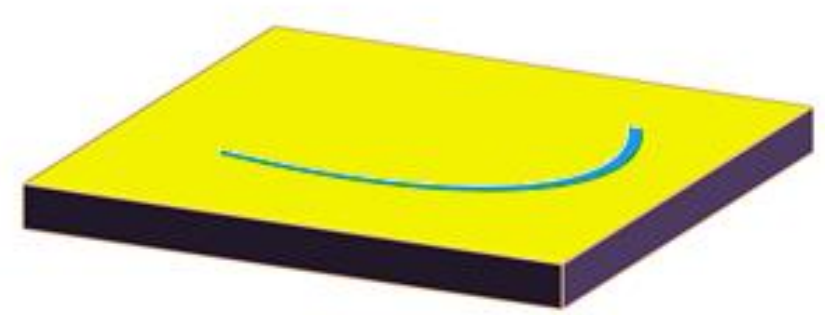

Fig. 2 Dynamic wettability of the as-prepared aligned nanorod arrays. (a-c) Oil is easy to roll on the aligned nanorod arrays surface in water with a sliding angle of $<5^{\circ}$. (d-f) Oil sticks to the smooth ZnO surface in water, and a droplet is difficult to move even with a higher sliding angle. Schematic illustration of the corresponding tri-phase contact line of the oil droplet on (g) the aligned nanorod arrays and on (h) a smooth $\mathrm{ZnO}$ surface in water. 

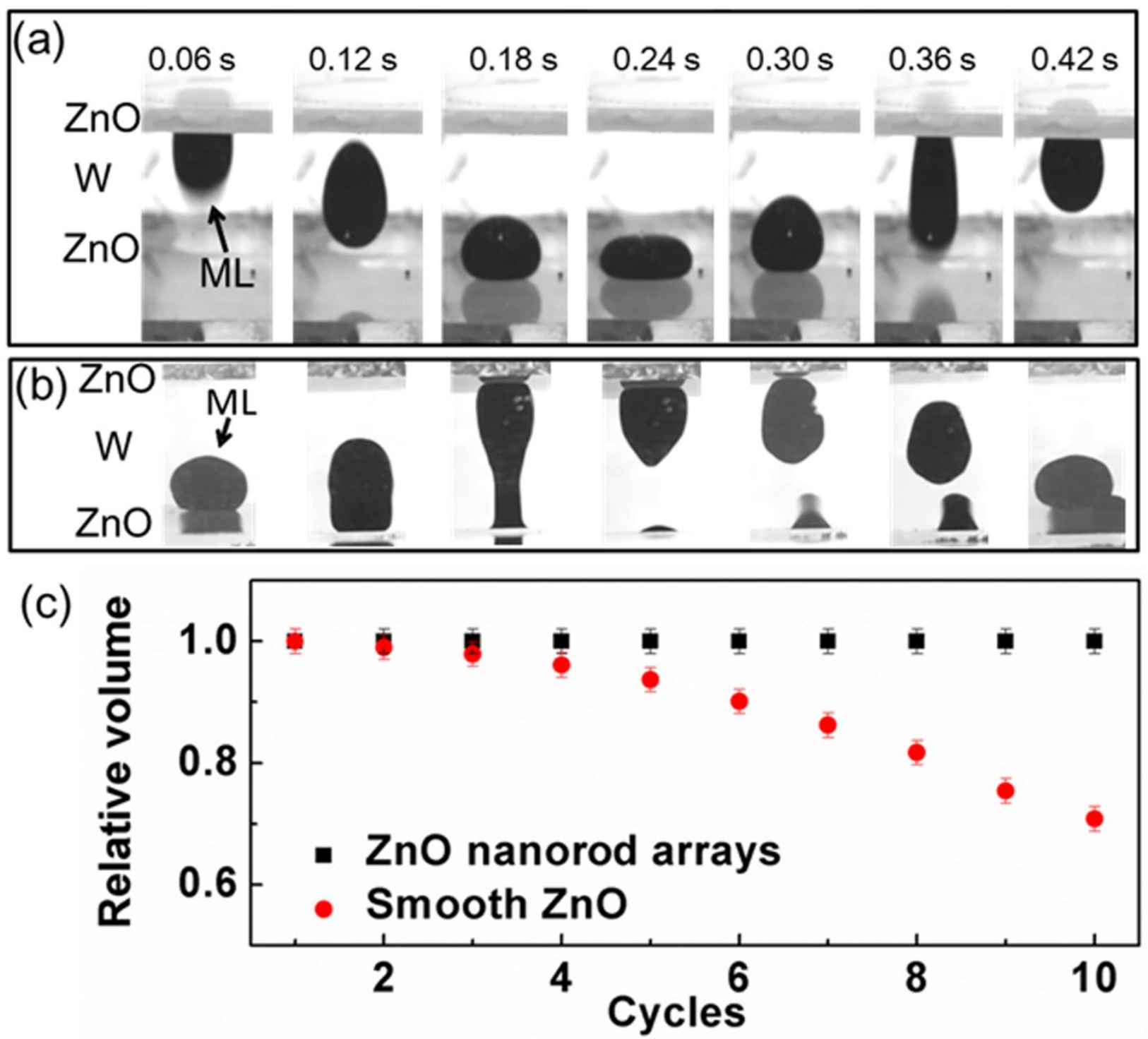

Fig. 3 The dynamic movement process of a magnetic fluid droplet on the aligned nanoarray and smooth ZnO substrates. (a) The dynamic movement process for a magnetic fluid droplet on an aligned ZnO nanorod arrays substrate with low adhesion. (b) The dynamic movement process of a magnetic fluid droplet on a smooth ZnO substrate with high adhesion. (c) The change in the relative volume of the magnetic fluid droplet on the bottom substrate during the movement for the aligned $\mathrm{ZnO}$ nanorod arrays and the smooth $\mathrm{ZnO}$ substrates. 

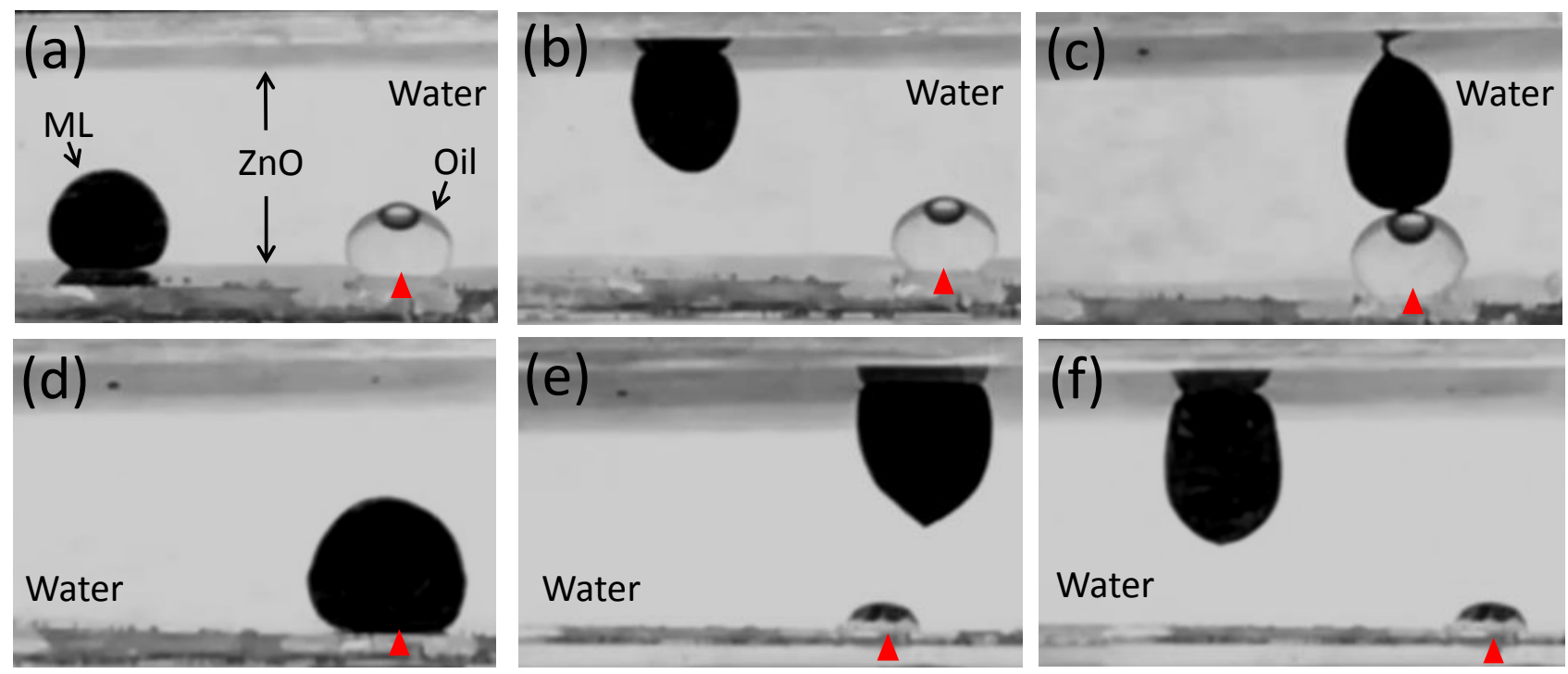

Fig. 4 The manipulation and transfer of an underwater oil droplet by a magnetic fluid droplet on the aligned ZnO nanorod arrays. (a-c) The magnetic fluid droplet movement and transfer process. (d-f) The magnetic fluid droplet combines with the oil droplet with a following movement and transfer process. The red triangle in each figure is the oleophilic area for fixing the droplet.

\section{A table of contents entry}

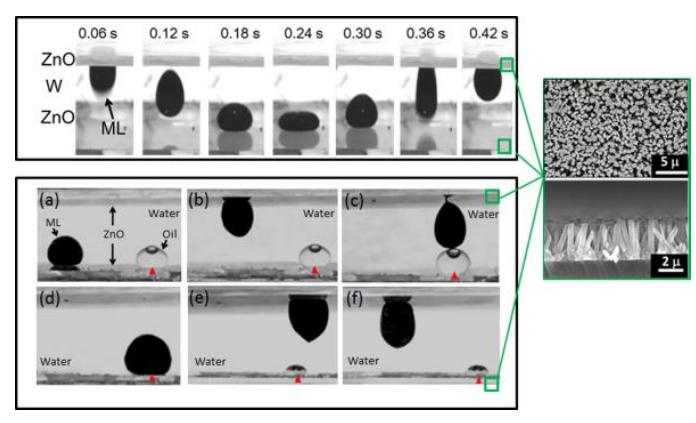

The magnetic-field-driven manipulation and transport of oil-based magnetic fluids are demonstrated underwater by using tri-phase systems. 